\title{
A Basic Study on Temporal Parameter Estimation of Wheelchair Propulsion based on Measurement of Upper Limb Movements Using Inertial Sensors
}

\author{
Takashi Watanabe*1, Kodai Miyazaki ${ }^{* 2}$, Maho Shiotani*1, \\ Andrew Symonds*3, Catherine Holloway*4, Tatsuto Suzuki ${ }^{*}$ \\ ${ }^{* 1}$ Graduate School of Biomedical Engineering, Tohoku University, Sendai, Japan \\ ${ }^{* 2}$ Graduate School of Engineering, Tohoku University, Sendai, Japan \\ ${ }^{*}$ Centre for Rehabilitation Engineering and Assistive Technology, University College London, London, United \\ Kingdom \\ ${ }^{* 4}$ Department of Computer Science, University College London, London, United Kingdom \\ ${ }^{* 5}$ Department of Civil, Environmental \& Geomatic Engineering, University College London, London, United Kingdom \\ e-mail addresses of all authors
}

\begin{abstract}
Wheelchairs are the most widely used assistive device to aid activities of daily living (ADL) for disabled people. However, manual pushing of a wheelchair frequently leads to overuse of upper extremities causing shoulder pain and carpal tunnel syndrome. The purpose of this study was to test a novel method of estimating temporal parameters of wheelchair propulsion using inertial sensors. In this paper, normalized coordinate values of the vector defined on the upper arm were calculated from an inertial sensor attached on the upper arm. The number of strokes and push cycle timings including duration of propulsion and recovery phases were estimated for steady state wheelchair propulsion. This estimation was completed using a novel vector-based approach and a previously published resultant acceleration method; both were compared to timings measured using the SmartWheel. Measurements were performed on level and sloped surfaces with 10 able bodied subjects. The vector-based method improved estimation of the number of strokes when compared to the resultant acceleration method. However, the push cycle was estimated with better accuracy by the resultant acceleration method. Therefore, a combination of the vector-based and the resultant acceleration methods is proposed to ensure more accurate estimation of temporal parameters. The results suggest inertial sensors can be used to measure wheelchair activity accurately and reliably.
\end{abstract}

\section{INTRODUCTION}

Manual wheelchairs are widely used as assistive devices to aid activities of daily living (ADL) for dsabled people. Wheelchairs have been shown to increase the quality of life of disabled people [1], but in doing so they put large loads on the upper limbs which subsequently have been shown to cause injury. For example, about $60 \%$ of the wheelchair users experienced shoulder pain [2-4] and more than $50 \%$ of the users had carpal tunnel syndrome [5]. These injuries have been shown to have to further reduce people's quality of life (QOL) due to increased pain and reduction in functional activities and therefore independence [6].

Such injuries of the upper limbs that are caused by wheelchair propulsion are considered to be associated with amplitude of force applied to the hand rim and the cycle of force application [7]. Therefore, it is recommended to reduce the number of strokes and to maximize the push arc and recovery time between pushes for wheelchair users [8]. Therefore being able to accurately measure the temporal paramters of pusing is very important to assessing the risk of upper limb injury.

The number of strokes and the temporal parameters of the push cycle during wheelchair propulsion can be quantitatively evaluated using an optical threedimensional motion analysis system and a force sesing handrim such as the SmartWheel [7, 9, 10]. This method makes it possible to measure movements and applied force with high accuracy. However, the high cost of such measurement tools make the cost prohibitive for many clincal settings. Further, there are few studies which have assessed pushing in non-clinical environments. In practical environments, higher push forces are applied when starting to push [11] and over everyday footway barriers [12]. Inertial sensors offer an opportunity to collect a greater range of data in outdoor environments and across a larger population due to the fact they are: not limited in measurement environment, easy-to-use, small in size, light weight and inexpensive.

Wheel-mounted accelerometers have been used to measure information on wheelchair movement [13]. In addition, Ojeda et al. [14] used triaxial accelerometers attached on the upper limb to estimate stroke number and temporal parameters of the push cycle [14]. This was completed using a method which necessitated determining trial-specific cutoff frequencies for the low-pass filter, which was applied to the acceleration signal. This might sometimes limit the monitoring of wheelchair users when it is expanded to a daily use.

We propose a new system for measuring the temporal parameters of pushing which would not require tuning for each run and a cutoff frequency of low-pass filter to remove high frequency noise of acceleration signals determined for each propulsion trial in order to be more applicable to everyay testing. This paper aimed at testing a method of estimating temporal parameters based on measurement of upper arm movements. Three 


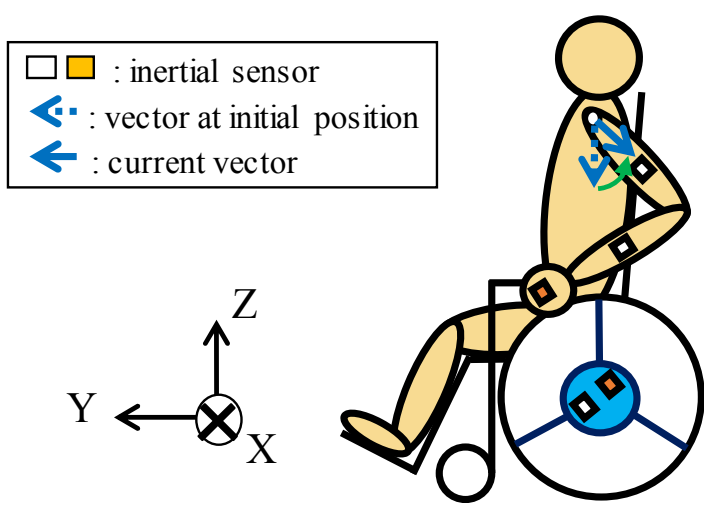

Figure 1 Outline of experimental setup and definition of vector of the upper arm.

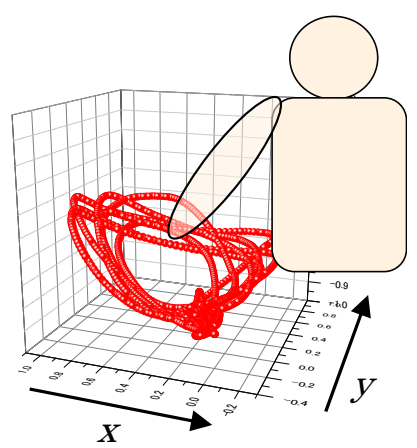

Figure 2 An example of measured locus of the vector defined on the upper arm during 3D movements of the shoulder joint.

dimensional upper arm movements were represented by the vector defined on the upper arm. In this paper, normalized coordinate values of the vector were calculated from signals measured with the inertial sensor attached on the upper arm.

The estimation method based on upper arm movements does not need to determine a cutoff frequency for the low pass filter for each trial as used in the previous study. Furthermore, there is a possibility to detect hand contact to the pushrim and hand release from it. It also makes possible to estimate propulsion and recovery phases. In this paper, a method of estimation of the number of strokes, the push cycle and detection of propulsion and recovery phases were examined for steady state propulsion on the level and sloped surfaces with nonwheelchair users.

\section{MEASUREMENT AND Estimation Methods}

\section{A. Measurement Method}

In this paper, signals measured with inertial senors attached on the left upper limb were used to estimate parameter values. As shown in Fig. 1, sensor signals during wheelchair propulsion were measured with inertial sensors attached on the upper arm, the forearm and the hand with stretch band or double sided tape. Inertial sensors were attached on the SmartWheel (left wheel) with double sided tape in order to synchronize signals of sensors and the SmartWheel.

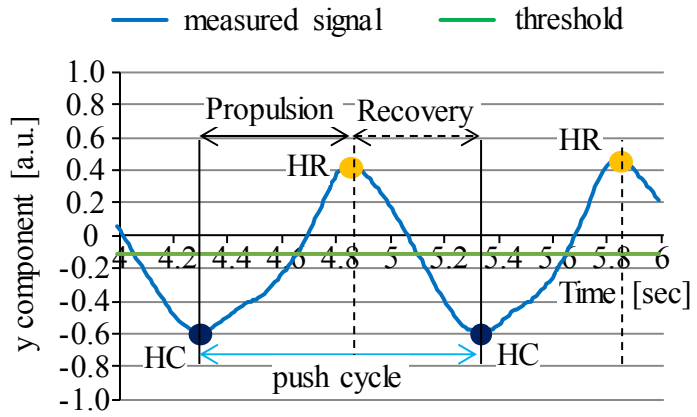

(a) vector-based method

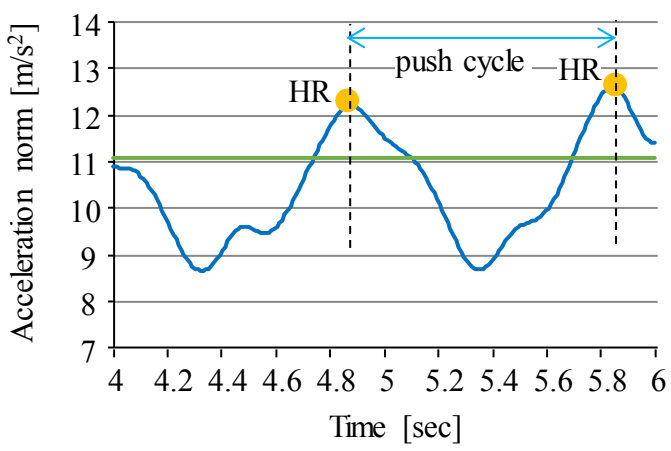

(b) Resultant acceleration method

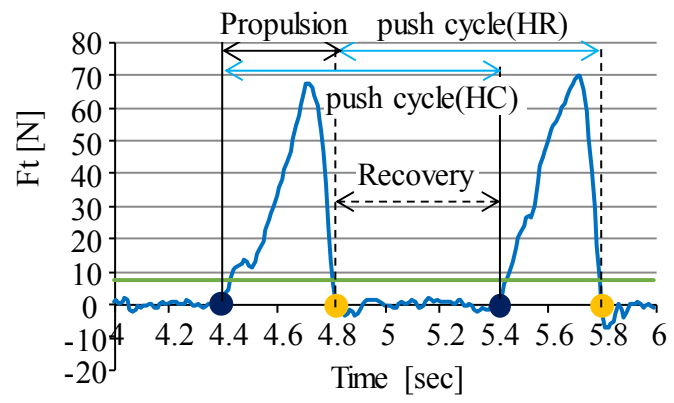

(c) SmartWheel

Figure 3 Definitions of parameters

\section{B. Vector-based Method}

A unit vector for the upper arm was defined in the longitudinal direction from the shoulder joint as the origin (Fig. 1). Normalized coordinate values of the vector can be calculated from the sensor signals on the upper arm based on 3 dimensional movement representation by using quaternion [15]. First, quaternion calculated from angular velocity was corrected by quaternion calculated from acceleration signals by Kalman filter. Then, a rotation matrix is calculated from the corrected quaternion. Rotation of the vector of the upper arm from the gravitational direction was calculated by using the rotation matrix. Figure 2 shows an example of locus of the vector, which is analogous to movement pattern of the elbow joint position. Since the y-component of the vector shows the forth and back direction, in this paper, push movements were detected by using the y-component of the vector.

An example of calculated value of y coordinate of the vector during wheelchair propulsion are shown in Fig. 3(a). First, a threshold is calculated for each trial as the mean value of the y-component of the vector within the 
steady state propulsion. Timing of hand contact (HC) on the pushrim was defined as the minimum value of past $y$ components preceded from the timing when the ycomponent was over the threshold. Timing of hand release (HR) from the pushrim was defined as the maximum value after the timing when the y-component was over the threshold. The stroke number was counted as the number of maximum points. Push cycle was estimated by time difference between two consecutive strokes using the $\mathrm{HC}$ timing or the HR timing.

\section{Resultant Acceleration-based Method}

The method using resultant accelerations based on Ojeda et al. [14] was implemented as follows. First, zerophase 2nd order Butterworth low pass filter was applied to the resultant accelerations measured with the inertial sensor. Figure 3(b) shows an example of the low-pass filtered resultant acceleration. Mean value of the low-pass filtered resultant accelerations was calculated within the steady state propulsion on each trial. The threshold value was determined as the mean acceleration plus 0.5 standard deviation.

Hand release (HR) from the pushrim was defined as peak value of the processed signal over the threshold. The stroke number was estimated by counting the detected HR. The push cycle was estimated by time difference between the HR timings of two consecutive strokes.

\section{Measurement of reference signals}

Reference signals for evaluation of estimated number of stroke, push cycle and propulsion and recovery phases were obtained from tangential force $(\mathrm{Ft})$ measured with the SmartWheel. Figure 3(c) shows an example of waveform of measured Ft signals. Timings of the HC on the pushrim and the HR from it were detected as the zero cross points of the $\mathrm{Ft}$ before and after reaching the threshold value $(7 \mathrm{~N})$, respectively.

The number of stroke was defined as the number of the HR timings and the push cycle was calculated as the time difference between two consecutive HR timings. The durations of propulsion and recovery phases were calculated between the $\mathrm{HC}$ and the HR and between the $\mathrm{HR}$ and the $\mathrm{HC}$, respectively.

\section{EXPERIMENTAL TEST}

\section{A. Experimental Method}

Ten able bodied subjects (i.e. non-wheelchair users) propelled the wheelchair on level surface of more than $7.2 \mathrm{~m}$ length and on sloped surface of about $4.8 \mathrm{~m}$ length with about $6 \%$ of incline with flat surface of more than $2 \mathrm{~m}$ length after the slope. Measurements were performed in 6 trials for each subject. Inertial sensors (WAA-010, Cresco wireless inc.) were attached on the upper arm, the forearm and the Smartwheel (left side of the wheel). Other inertial sensors (MTw, Xsens Technologies B.V.) were attached on the hand and the SmartWheel.

Sampling frequencies were $240 \mathrm{~Hz}$ and $100 \mathrm{~Hz}$ for the SmartWheel and inertial sensors, respectively. The signals measured with the SmartWheel were down sampled to $100 \mathrm{~Hz}$ before analysis. In this paper, push movements in the steady state propulsion were analyzed by removing the first stroke and the breaking movements.

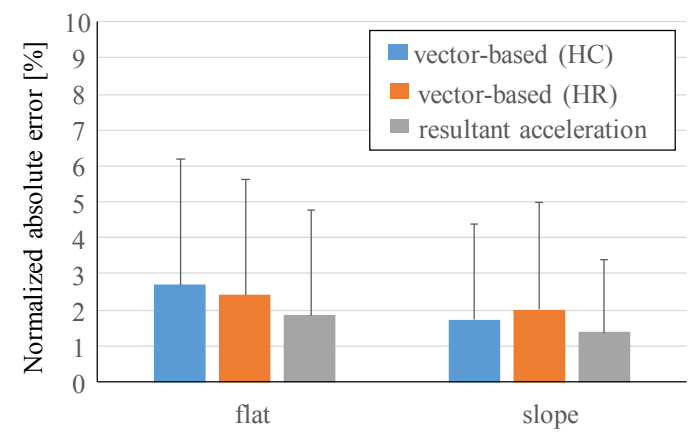

Figure 4 Normalized absolute error of push cycle estimation.

\section{B. Results}

Three measurement trials on flat surface and 2 trials on sloped surface were removed from analysis because of troubles of measurement devices in data recording. Totally, 408 and 442 strokes for flat and sloped surfaces obtained from 10 subjects were analyzed.

Before calculating parameter values, a cutoff frequency of low pass filter for the resultant accelerationbased method was determined as $2 \mathrm{~Hz}$ by analyzing the data of the 3rd trial of each subject. That is, the number of misdetection of the stroke was smallest with the cutoff frequency of $2 \mathrm{~Hz}$, in comparing the cutoff frequency ranging from 2 to $6 \mathrm{~Hz}$ that were used in the previous study [14]. Then, the resultant acceleration method was examined in estimation of the number of stroke with 3 inertial sensors for wheelchair propulsion on the flat surface. Misdetection of the stroke was smallest with the sensor attached on the upper arm $(2.5 \%)$. The senor on the hand showed the largest number of misdetection $(31.1 \%)$. The sensor on the forearm showed misdetection ratio between the upper arm sensor and the hand senor $(8.6 \%)$.

By using the upper arm senor, misdetection ratios were compared between the 2 estimation methods. For the flat surface, as described above, the resultant acceleration method caused 10 misdetections $(2.5 \%)$ with 4 subjects. One subject showed $17.6 \%$ misdetection. On the other hand, the vector-based method did not show misdetection. For the sloped surface, both methods caused misdetections: 2 misdetections $(0.5 \%)$ with 2 subjects with the vector-based method and 13 misdetections $(2.9 \%)$ with 3 subjects for the resultant acceleration method. One subject showed $38.1 \%$ misdetection with the resultant acceleration method.

Results of push cycle estimation are shown in Fig. 4. For the vector-based method, the push cycle was estimated by both the HC timing and the HR timing. For the both surface conditions, although the resultant acceleration method showed the smallest average values and variations of the error in estimation of push cycle, the both estimation methods could estimate the push cycle with good accuracy less than $3 \%$ in average.

The vector-based method can detect both the $\mathrm{HC}$ and the HR timings and estimate the propulsion and the recovery phases. The results are shown in Figs 5 and 6 . Although the time differences of the detected timings for the sloped surface were less than $50 \mathrm{~ms}$ in average, the differences for the propulsion on the level surface were 


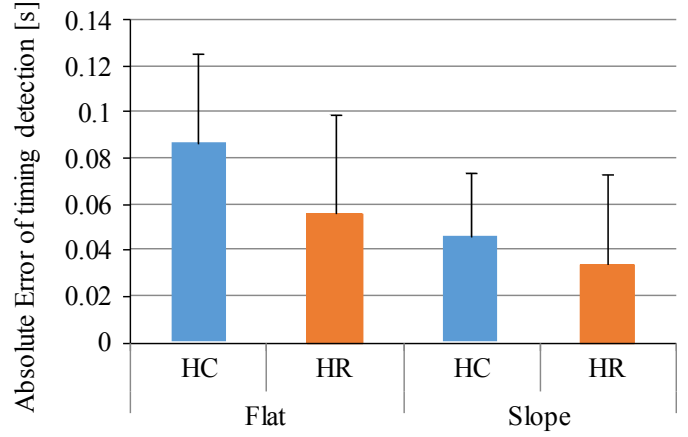

Figure 5 Time differences of detection of the HC and the HR timings by using the vector-based method with the inertial sensor on the upper arm.

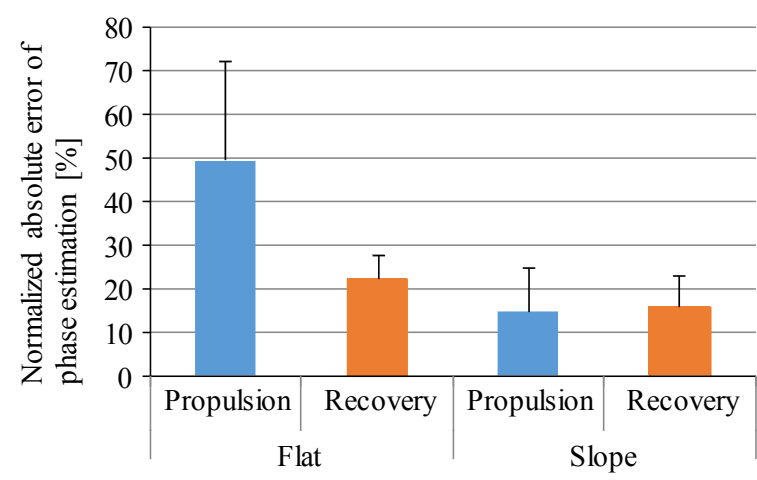

Figure 6 Normalized absolute error of estimation of propulsion and recovery phases with the vector-based method with the inertial sensor on the upper arm.

larger than the sloped surface. Errors of estimated propulsion and recovery phases were larger than $10 \%$ for both surfaces. Especially, propulsion phase on the flat surface was about $50 \%$ error in average.

\section{A Test OF a COMBined Estimaiton Method of THE PROPULSION AND THE RECOVERY PHASES}

As a possibility of improving the detection of the HC and the HR timings and the estimation of the propulsion and the recovery phases, a combination of the both estimation methods was examined. That is, the timings were detected by the resultant acceleration after the detection of stroke movement by the vector-based method. Here, the inertial sensor attached on the hand was used for the resultant acceleration method, because it was assumed that large acceleration was produced at the $\mathrm{HC}$ after backward movement and at the HR before forward movement. The propulsion and the recovery phases were estimated by using the $\mathrm{HC}$ and the HR timings detected by the combined method.

Results of detection of the $\mathrm{HC}$ and the $\mathrm{HR}$ and estimation of the phases for steady state strokes are shown in Figs. 7 and 8. Time differences of the $\mathrm{HC}$ and the HR timings from the reference signals were reduced to smaller values than those of the vector-based method. Estimation of both phases were also improved, although variation of the error was still large for the propulsion phase on the flat surface.

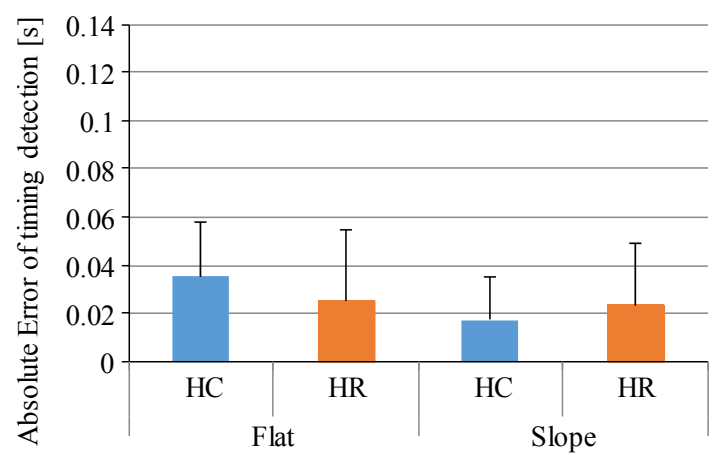

Figure 7 Time differences of detection of the HC and the HR timings by the combined method with inertial sensors on the upper arm and the hand.

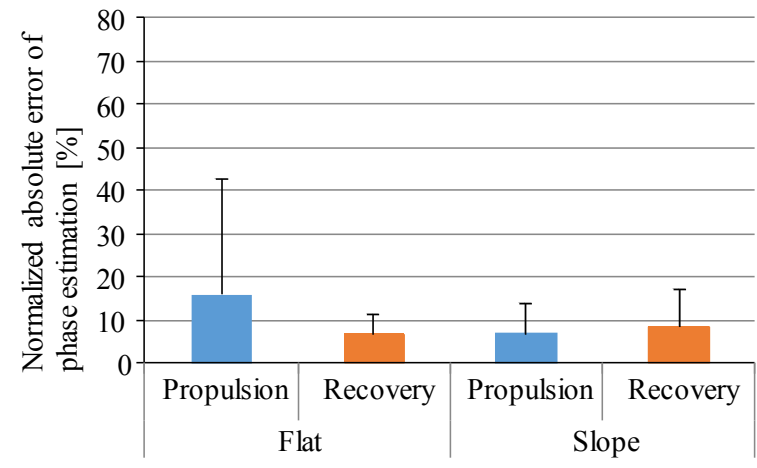

Figure 8 Normalized absolute error of estimation of propulsion and recovery phases with the combined method with inertial sensors on the upper arm and the hand.

\section{DISCUSSIONS}

Misdetection of strokes during wheelchair propulsion movements were improved by the vector-based method proposed in this study. The misdetection of the vectorbased method was caused with 2 subjects on the sloped surface, which were 1 of 65 strokes and 1 of 60 strokes, respectively. Although average misdetection rate of the resultant acceleration method was not so large, the method caused large misdetection rate with some subjects, which were 3 of 38,3 of 43 and 3 of 17 strokes on the flat surface, and 8 of 21 and 4 of 43 strokes on the sloped surface.

For estimation of the push cycle, the resultant acceleration method showed better estimation. This was because some subjects produced large movements before the $\mathrm{HC}$ and/or after the HR, which caused large error in the vector-based method. The resultant acceleration method is necessary to examine further in daily use of wheelchair, because a cutoff frequency have to be determined for each trial. The resultant acceleration method is currently considered to be useful for laboratory tests under the regulated experimental conditions.

The detection method of the $\mathrm{HC}$ and the HR were modified by assuming that large acceleration is produced at the $\mathrm{HC}$ and the $\mathrm{HR}$ timings. Although the resultant acceleration can be useful to estimate the timings, it sometimes caused large misdetection rate of the stroke. Large variation and average value of error for the propulsion phase was because large acceleration was not 
produced at the HC with some strokes. Since the combination of acceleration data and movement of upper limb would be effective, further improvement of the method would be expected.

In this paper, strokes during wheelchair movements were analyzed excluding the first stroke and the braking motions, because of preliminary tests. In addition, for the resultant acceleration method, a cut off frequency of $2 \mathrm{~Hz}$ was used after a preliminary test of stroke detection. This was because the previous study used the cut off frequency between 2 and $6 \mathrm{~Hz}$. Since the results of this paper showed that inertial sensors were suggested o be used to measure wheelchair activity accurately and reliably, the method is expected to be modified for practical, daily use decreasing the number of sensors.

\section{CONCLUSION}

The vector-based method proposed in this paper improved detection of strokes during wheelchair propulsion. The method also made it possible to detect hand contact and hand release timings, and estimation of propulsion and recovery phases. A combination of the vector-based and the resultant acceleration methods were shown to improve accuracy of detection of the $\mathrm{HC}$ and the HR timings and estimation of the propulsion and the recovery phases. The results suggest inertial sensors can be used to measure wheelchair activity accurately and reliably.

\section{REFERENCES}

[1] R. Devitt, B. Chau, and J. W. Jutai, "The Effect of Wheelchair Use on the Quality of Life of Persons with Multiple Sclerosis," Occup. Ther. Health Care, vol. 17, no. 3-4, pp. 63-79, Jan. 2004

[2] D. D. Gutierrez, L. Thompson, B. Kemp, and S. J. Mulroy, "The Relationship of Shoulder Pain Intensity to Quality of Life, Physical Activity, and Community Participation in Persons with Paraplegia," J. Spinal. Cord Med., vol. 30, no. 3, pp. 251-255, 2007.

[3] M. Alm, H. Saraste, and C. Nobbrink, "Shoulder pain in persons with thoracic spinal cord injury: prevalence and charactristics," $J$ Rehabil. Med., vol. 40, no. 4, pp. 277-283, April 2008.

[4] K. A. Curtis, G. A. Drysdale, R. D. Lanza, M. Kolber, R. S. Vitolo, and R. West, "Shoulder Pain in Wheelchair Users with Tetraplegia and Paraplegia," Arch. Phys. Med. Rehabil., vol. 80, no. 4, pp. 453-457, April 1999.

[5] J. Yang, M. L. Boninger, J. D. Leath, S. G. Fitzgerald, T. A Dyson-Hudson, and M. W. Chang, "Carpal Tunnel Syndrome in Manual Wheelchair Users with Spinal Cord Injury A CrossSectional Multicenter Study," Am. J. Phys. Med. Rehabil., vol. 88, no. 12, pp. 1007-1016, December 2009.

[6] D. D. Gutierrez, L. Thompson, B. Kemp, S. J. Mulroy, “ The Relationship of Shoulder Pain Intensity to Quality of Life, Physical Activity, and Community Participation in Persons With Paraplegia," J. Spinal Cord Med., vol. 30, no. 3, pp. 251-255, 2007.

[7] M. L. Boninger, B. G. Impink, R. A. Cooper, and A. M. Koontz, "Relation Between Median and Ulnar Nerve Function and Wrist Kinematics During Wheelchair Propulsion," Arch. Phys. Med. Rehabil., vol. 85, no. 7, pp. 1141-1145, July 2004.

[8] Paralyzed Veterans of America Consortium for Spinal Cord Medicine, "Preservation of upper limb function following spinal cord injury: a clinical practice guideline for health-care professionals," J. Spinal cord Med., vol. 28, no. 5, pp. 434-470, 2005.

[9] A. M. Koontz, R. A. Cooper, M. L. Boninger, A.L. Souza, B. T. Fay, "Shoulder kinematics and kinetics during two speeds of wheelchair propulsion," J. Rehabil. Res. Dev., vol. 39, no. 6, pp. 635-649, 2002.

[10] SmartWheel, http://www.out-front.com/smartwheel_overview.php (April 20, 2016)

[11] A. M. Koontz, R. A. Cooper, M. L. Boninger, Y. Yang, B. G. Impink, and L. H. van der Woude, "A kinetic analysis of manual wheelchair propulsion during start-up on select indoor and outdoor surfaces," J. Rehabil. Res. Dev., vol. 42, no. 4, pp. 447-458, 2005

[12] C. S. Holloway, A. Symonds, T. Suzuki, A. Gall, P. Smitham, and S. Taylor, "Linking wheelchair kinetics to glenohumeral joint demand during everyday accessibility activities," Proc. 37th Annual Int. Conf. IEEE EMBS, pp. 2478-2481, 2015.

[13] E. H. Coulter, P. M. Dall, L. Rochester, J. P. Hasler, M. H. Granat, "Development and validation of a physical activity monitor for use on a wheelchair," Spinal Cord, vol. 49, no.3 pp. 445-450, 2011

[14] M. Ojeda and D. Ding, "Temporal Parameters Estimation for Wheelchair Propulsion Using Wearable Sensors," Biomed Res. Int., vol. 2014, Article ID 645284, 10pages, 2014.

[15] M. Shiotani, T. Watanabe, "A Preliminary Study on Evaluation of circumduction movement during gait with Wireless Inertial Sensors," Proc. 36th Annual Int. Conf. IEEE EMBS, pp. 58285831, 2014. 